\title{
Asymptotic Giant Branch Stars and presolar grains
}

\author{
Maurizio Busso ${ }^{1,3, *}$, Sara Palmerini ${ }^{1,3, * *}$, and Diego Vescovi ${ }^{2,3,4, * * *}$ \\ ${ }^{1}$ Department of Physics and Geology, via A. Pascoli snc, 06123 Perugia \\ ${ }^{2}$ Gran Sasso Science Institute, Via F. Crispi 7, 67100 L'Aquila \\ ${ }^{3}$ INFN, Section of Perugia, via A. Pascoli snc, 06123 Perugia \\ ${ }^{4}$ INAF, Observatory of Abruzzo, via Mentore Maggini snc, 64100 Teramo
}

\begin{abstract}
Starting from the recognition that radioactive isotopes were present alive in the Early Solar System, inducing composition anomalies from their decay, and through the discovery that other important anomalies affected also stable species, we shall discuss how the carriers of these abundance peculiarities were identified in very refractory pre-solar dust grains, formed in circumstellar environments. We shall outline how groups of such grains and subsequently individual single crystals of $\mathrm{C}$-rich or O-rich materials (like, e.g., $\mathrm{SiC}$ and $\mathrm{Al}_{2} \mathrm{O}_{3}$ ) could be analyzed, providing a new tool to verify the composition of stellar winds. This is so especially for AGB stars, which are the primary factories of dust in the Galaxy. For this reason, pristine meteorites open a crucial window on the details of nucleosynthesis processes occurring in such evolved red giants, for both intermediate-mass elements and rare heavy nuclei affected by slow neutron captures (the s-process).
\end{abstract}

\section{Introduction}

Along the past 60 years, starting with the identification in pristine meteorites of an excess in ${ }^{129} \mathrm{Xe}$ from the in situ decay of ${ }^{129} \mathrm{I}$ [1], we have known that the average Early Solar System (ESS) composition was contaminated through the addition of various materials coming from other stars and not previously homogenized in the Interstellar Medium (ISM) [2]. Discovery of anomalous abundances in certain isotopes that are daughters of radioactive nuclei involved several elements in the last decades of the 20th century [3,4]. Among other amazing findings, they led to the realization that the $n$-deficient isotope of $\mathrm{Al},{ }^{26} \mathrm{Al}$, had been present in the ESS in a huge and uniform concentration $\left({ }^{26} \mathrm{Al} /{ }^{27} \mathrm{Al} \simeq 5 \cdot 10^{-5}\right)$, sufficient to affect significantly, or even control, the heating of ancient materials, which induced the early differentiation of planetary embryos $[5,6]$. The presence in the ESS of live radioactive isotopes from various stellar sources (whose decay products, signalling their earlier existence, are often called fossil radioactivities) was since then the object of intense research [7-10].

The specific way in which such contaminations were possible was through the transport, across the ISM, of very refractory solid particles condensed around cool stars [11], capable of surviving the thermal stresses of many Galactic and Star-Formation processes. Such refractory dust grains can be produced in the circumstellar envelopes of evolved stars and are

\footnotetext{
*e-mail: maurizio.busso@pg.infn.it

**e-mail: sara.palmerini@pg.infn.it

***e-mail: diego.vescovi@gssi.it
} 
therefore expected to carry the signature of various nucleosynthetic processes, involving both major species and trace elements [12]. The identification and collection of samples enriched in such grains (especially $\mathrm{SiC}$ ) were then made possible by improved mass-spectrometry facilities and innovative separation techniques, made by sequential acid treatments of materials from pristine meteorites [13]. Their analysis soon revealed that they were the carriers of a large excess in ${ }^{22} \mathrm{Ne}$, the so-called $\mathrm{Ne}-\mathrm{E}(\mathrm{H})$ anomaly [14], which was subsequently recognized as being produced in the thermonuclear $\mathrm{H}$ - and He-shells of moderately massive stars (See Figure 1 and $[15,16]$ ), during their so-called Asymptotic Giant Branch (AGB) phases. These evolutionary stages follow the exaustion of $\mathrm{He}$ in the central core, which is therefore formed of $\mathrm{C}$ and $\mathrm{O}$; it undergoes strong electron degeneracy and will be left as a compact remnant (a white dwarf) at the end of the evolution. Above the core, two thermonuclear shells of $\mathrm{He}$ and $\mathrm{H}$ burn alternatively, while the intershell region is swept repeatedly by intermediate convective instabilities (from now on called Intermediate Convective Shells, ICS).

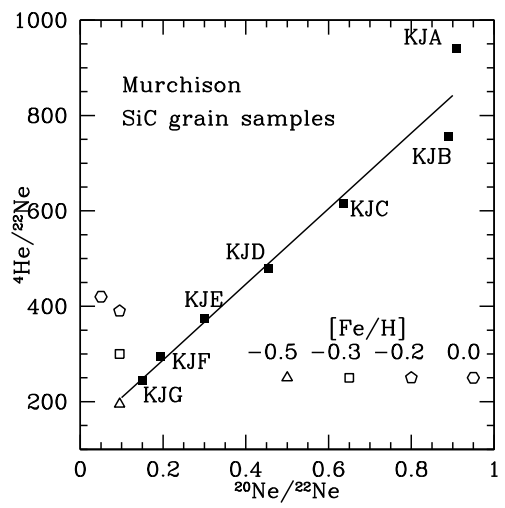

Figure 1. Isotopic ratios of light noble gases in $\mathrm{SiC}$ grain admixtures from the Murchison chondrite (filled squares) versus model predictions of the He-rich layers in AGB stellar models (open symbols). The data indicate the presence in the samples of a very large anomaly $(\mathrm{Ne}-\mathrm{E}(\mathrm{H}))$ with respect to the average solar system ratios, which are: $\left({ }^{4} \mathrm{He} /{ }^{22} \mathrm{Ne}\right)_{\odot}=1.18 \times 10^{4} ;\left({ }^{20} \mathrm{Ne} / 22 \mathrm{Ne}\right)_{\odot}=13.67$ [17]. AGB stars of lower-than-solar composition were shown by [15] to account for the abundances in the KJG samples, those more heavily treated with acids (hence cleaner from solar-system contaminations). The other samples lay on a linear mixing sequence between purely stellar and solar compositions.

\section{An AGB origin for meteoritic anomalies}

Inside the He-rich layers present in the above-mentioned AGB phases (occurring in stars below a stellar mass of about $8 \mathrm{M}_{\odot}$ ), the advancing H-burning shell, proceeding through $\mathrm{CNO}$ cycling, leaves behind an enhanced ${ }^{14} \mathrm{~N}$ abundance (up to a concentration of 0.01 by mass, for a solar-like composition). This occurs in a zone that is gradually compressed and heated, as its base cannot adjust, being in contact with the inert, degenerate $\mathrm{CO}$ core. Due to this, $\mathrm{He}$ is forced to ignite semi-explosively (at a temperature reaching a peak of up to $3-3.5 \times 10^{8} \mathrm{~K}$, depending on the mass) and ${ }^{14} \mathrm{~N}$ undergoes a double $\alpha$-capture, producing the excess ${ }^{22} \mathrm{Ne}$ that accounts for the $\mathrm{Ne}-\mathrm{E}(\mathrm{H})$ anomaly in presolar meteoritic materials. The peculiar changes of the stellar structure occurring in these stages are shown in Figure 2. The plot shows the position of the H-burning shell, $\mathrm{M}_{\mathrm{H}}$ and of the inner border of the envelope convection, $\mathrm{M}_{\mathrm{CE}}$. This last has a sudden increase corresponding to the mentioned temperature peak of the Heshell (thermal pulse), which is accompanied by the development of the ICS mentioned in the 
text (shaded in the figure). The ICS brings to the top of the He layers the products of fast He burning occurring at the bottom (not shown). Subsequently, $\mathbf{M}_{\mathrm{CE}}$ penetrates the layers previously involved in the ICS, through a process called third dredge up (TDU), carrying freshly synthesized nuclei to the surface. The H-He discontinuity (estinguished $\mathrm{H}$ shell) correspondingly recedes for a few thousand years, before H-burning restarts and the cycle repeats.

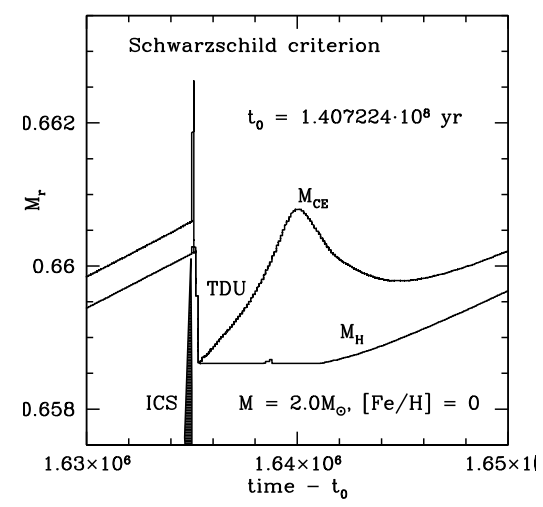

Figure 2. An example of the thermal-pulse and TDU sequence in a low mass AGB star of solar metallicity. It is the nineth cycle of this type in the model star. ${ }^{14} \mathrm{~N}$ from $\mathrm{CNO}$ processing is present below the H-burning shell, before the onset of the ICS; in the intermediate convective region it becomes ${ }^{22} \mathrm{Ne}$ through a double $\alpha$ capture. This last burns only marginally, releasing modest neutron fluxes through ${ }^{22} \mathrm{Ne}(\alpha, \mathrm{n})^{25} \mathrm{Mg}$. Most of the neutrons for $s$-processing are however released below the TDU, following first the penetration of protons, then the reaction ${ }^{12} \mathrm{C}(\mathrm{p}, \gamma){ }^{13} \mathrm{C}$, and finally the ${ }^{13} \mathrm{C}(\alpha, \mathrm{n}){ }^{16} \mathrm{O}$ reaction.

\section{Deep mixing and s-processing as constrained by presolar grains}

The same existence of the $\mathrm{Ne}$ anomaly shows that the ${ }^{22} \mathrm{Ne}$ produced from ${ }^{14} \mathrm{~N}$ combustion is not consumed during the rapid thermonuclear processes accompanying the development of the ICS. This fact has important consequences, as we shall now discuss.

After the early discovery of live Tc in their photospheres [18] and the theoretical work done by several groups in the seventies (see e.g. [19]), AGB stars are known to be the site where the bulk (the main component) of heavy nuclei near the valley of $\beta$-stability are produced, through slow neutron captures (the s-process). For decades this was attributed to the release of neutron fluxes by the ${ }^{22} \mathrm{Ne}(\alpha, \mathrm{n})^{25} \mathrm{Mg}$ reaction inside the above mentioned ICSs [20]; however, the explanation of the anomalous Ne composition of grains matched previous results of stellar spectroscopy [22] in indicating that ${ }^{22} \mathrm{Ne}$ actually does not burn efficiently. Its survival requires that the stars where the anomaly is generated and where $s$-processing occurs be of low mass $\left(\mathrm{M} / \mathrm{M}_{\odot} \lesssim 3\right)$, because in this case the maximum temperature of the ICS does not exceed $3 \times 10^{8} \mathrm{~K}$ (a value for which ${ }^{22} \mathrm{Ne}$ burns only marginally). This also requires that another neutron source, the reaction ${ }^{13} \mathrm{C}(\alpha, \mathrm{n}){ }^{16} \mathrm{O}$, controls neutron captures in AGB stars.

However it became clear, in many years of research, that producing locally fresh ${ }^{13} \mathrm{C}$ requires the ingestion of protons below the $\mathrm{H}-\mathrm{He}$ interface at TDU [23], a fact not predicted by standard models adopting the Schwarzschild criterion for convection (Figure 2). This 
implies that low mass AGB stars must be affected by extra-mixing phenomena producing the neutron source (see [10]).

In fact, extra-mixing phenomena are known to occur in evolved low mass stars both during the Red Giant Branch (RGB) and during the AGB phases (we refer to [21] for details). Again, presolar grains (in this case mainly corundum, i.e. $\mathrm{Al}_{2} \mathrm{O}_{3}$ ) provided crucial clues for the required stellar physics improvements (see Figure 3).

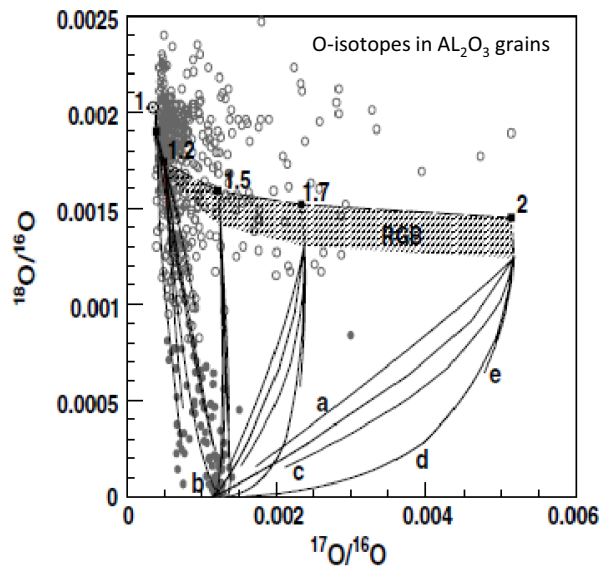

Figure 3. Isotopic anomalies in presolar grains of $\mathrm{Al}_{2} \mathrm{O}_{3}$, recovered from pristine meteorites, versus predictions of stellar models. The abundance sequence expected by the standard evolution of RGB stars from 1 to $2 \mathrm{M}_{\odot}$ does not reproduce the presolar grain evidence, especially for grains more heavily depleted in ${ }^{18} \mathrm{O}$. They are however accounted for by models assuming slow, extended mixing along the RGB and AGB phases. This is shown by the model curves extending downwards from the RGB region. They represent the isotopic abundances achieved assuming extra-mixing at different speeds (progressively slower from a to e) for different initial masses. This also shows that most ${ }^{18} \mathrm{O}$-depleted corundum grainds descend from very low mass stars (left side of the figure). See also [21]; the figure is adapted from that paper.

Not only for the isotopes of major elements, like oxygen, but also for those of rare trace species, like those heavier than $\mathrm{Fe}$, the constraints from presolar materials had in the mean time become available from single grains, extracted and analyzed through several innovative techniques. In particular, we mention the new generation of mass spectrometry instruments based on laser-induced ionization of vapours obtained by sputtering [27].

New attempts were also presented, aimed at modelling quantitatively extramixing phenomena suitable to explain the isotopic admixture of oxygen (and carbon) in advanced evolutionary phases of low mass stars. We mention, in particular, those invoking diffusive processes (like thermohaline mixing, see [24]) and those based on more rapid, oscillatory or wave-like mechanisms. These last were soon revealed to be much more effective $[25,26]$ and actually offer now the most widely debated scenarios. These same models also provide estimates for the extension of the ${ }^{13} \mathrm{C}$ reservoir (or pocket, as it is often called), where s-process nucleosynthesis occurs.

In particular, based on previous work by [25], Palmerini et al. [28] were recently able to show that the new generation of $s$-process models, based on the rather peculiar ${ }^{13} \mathrm{C}$ pockets formed by MHD-mixing in AGB stars, now permit, for the first time and in a non-parametric 


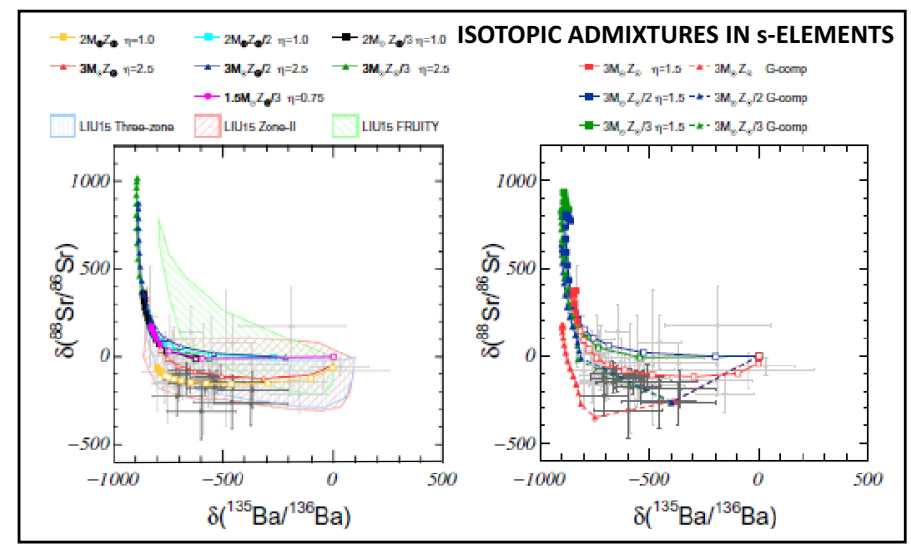

Figure 4. Examples of the agreement achieved between $s$-process models with MHD-induced ${ }^{13} \mathrm{C}$ reservoirs and the record of trace-element isotopic ratios in single $\mathrm{SiC}$ grains. The light dots with error bars represent the measurements; the heavy dots and the curves are from models of the indicated stellar masses, with mass loss estimated from the Reimers' parameterization (its efficiency is characterized by the $\eta$ value shown). The term "G-component" refers to the material cumulatively mixed to the envelope from the He-intershell, without dilution with envelope material (see [28] for details).

way, to reproduce accurately the isotopic measurements in single grains of presolar SiC provided by the mentioned new experimental techniques [27].

\section{References}

[1] Reynolds, J.H. 1960, Phys. Rev. Lett. 4, 351

[2] Huss, G.R. \& Lewis, R.S. 1995, Geochim. Cosmochim. Acta 59, 115

[3] Cameron, A.G.W. 1993, in Protostars and Planets III, ed. E.H. Levy, J.L. Lunine, (Tucson: Univ. Arizona Press) p. 47

[4] Busso, M. 2019, The Early Solar System, in Astronomy with Radioctivities, Ed...

[5] Lee, T., Papanastassiou, D.A., \& Wasserburg, G.J. 1977, Ap. J. 211, 107

[6] Wasserburg, G.J. 2003, Ann. Rev. of Earth \& Plan. Sci. 31, 1

[7] Truran, J. \& Cameron, A.G.W. 1978, Ap J. 219, 226

[8] Wasserburg, G.J, Busso, M., Gallinio, R. 1994, Ap. J.

[9] Wasserburg, G.J, Busso, M., Gallinio, R. 1995, Ap. J.

[10] Busso, M., Gallino, R., \& Wasserburg, G.J. 1999, Ann. Rev. Astron. Astrophys. 37, 239

[11] Lodders, C., Fegley, B. 1995, Meteoritics, 30, 661

[12] Anders, E. \& Zinner, E. 1993, Meteoritics 28, 490

[13] Amari, S., Lewis, R.S. \& Anders, E. 1991, Metoritics 26, 314

[14] Tang, M. \& Anders, E. 1988, Geochim. Cosmochim. Acta 52,1235

[15] Gallino, R., Busso, M., Picchio, G. \& Raiteri, C.M. 1990, Nature 348, 298

[16] Lewis, R.S., Amari, S., \& Anders, E. 1990, Nature 348, 293

[17] Lodders, K., \& Palme, H. 2009, M\&PSA 72, 515 
[18] Merrill, P W. 1952, Scienze 115, 484

[19] Iben, I. jr. \& Renzini, A. 1983 Ann. Rev. Astron. Astrophys. 21, 271

[20] Iben, I.jr. \& Truran, J.W. 1978, Ap J 220, 980

[21] Palmerini, S., La Cognata, M. Cirstallo, S. \& Busso, M. 2011, Ap. J. 729, 3

[22] Smith, V. \& Lambert, D.L. 1986, ApJ 311, 843

[23] Iben, I. jr. \& Renzini, A. 1982, ApJ 249, L79

[24] Eggleton, P. P., Dearborn, S. P. \& Lattanzio, J. C. 2006, Science 314, 1580

[25] Trippella, O., Busso, M., Palmerini, S. et al. 2016, ApJ 818, 125

[26] Battino, U., Tattersall, A., Lederer-Woods, C. et al. 2019, MNRAS 489, 1082

[27] Liu, N., Savina, M., Gallno, R. et al. 2015, ApJ 803, 12

[28] Palmerini, S., Tripella, O. Busso, M., et al. 2018, Geochim Cosmochim Acta 221, 21 